\title{
Application of the Multiplex PCR Method for Discrimination of Artemisia iwayomogi from Other Artemisia Herbs
}

\author{
Mi Young LeE, ${ }^{a, \#}$ Eui Jeong DoH,${ }^{b, \#}$ Eung Soo KIM, ${ }^{b, 1)}$ Young Wha KIM, ${ }^{a}$ Byong Seob Ko, ${ }^{a}$ and \\ Seung-Eun $\mathrm{OH}^{*, b}$ \\ ${ }^{a}$ Korea Institute of Oriental Medicine; Deajeon 305-811, Republic of Korea; and ${ }^{b}$ Department of Biological Sciences, \\ Konkuk University; Seoul 143-701, Republic of Korea. \\ Received August 7, 2007; accepted January 16, 2008; published online January 18, 2008
}

\begin{abstract}
Some plants classified in the genus Artemisia are used for medicinal purposes. In particular, A. iwayomogi, which is referred to as 'Haninjin,' is used as an important medicinal material in traditional Korean medicine. However, $A$. capillaris, and both $A$. argyi and $A$. princeps, referred to as 'Injinho' and 'Aeyup', respectively, are used for purposes other than those for which 'Haninjin' is utilized. However, it is occasionally difficult to differentiate 'Haninjin' from 'Injinho' and/or 'Aeyup' on the basis of their morphological features, particularly when in the dried and/or sliced form. Therefore, the development of a reliable method by which to discriminate 'Haninjin' from other Artemisia herbs, especially 'Injinho' and 'Aeyup', is clearly necessary. We recently determined that the RAPD (random amplified polymorphic DNA) technique can be used to discriminate efficiently between some Artemisia herbs. In particular, when applied to RAPD, the non-specific UBC primer 391 (5'-GCG AAC CTC G-3') was demonstrated to amplify PCR products specific to $A$. iwayomogi. Based on the nucleotide sequences of the PCR product, we designed a 2 F1 (5'-ACC TCG GAC CTA AAT ACA-3')/ 2 F3 (5' ${ }^{\prime}$-TTA TGA TTC ATG TTC AAT TC-3') primer set to amplify a SCAR (sequence-characterized amplified region) marker of $A$. iwayomogi. Employing this primer set, along with two other primer sets amplifying SCAR markers of 'Aeyup' (A. argyi and $A$. princeps) and both 'Injinho' ( $A$. capillaris) and $A$. japonica, which are classified into the same subgroup in a phenogram constructed from RAPD analysis, we developed a multiplex PCR method by which $A$. iwayomogi could be discriminated with certainty from other Artemisia herbs. Via this method, we determined not only whether the tested Artemisia herb was $A$. iwayomogi, but also which Artemisia herbs were tested concurrently with $A$. iwayomogi.
\end{abstract}

Key words Artemisia iwayomogi; Artemisia herb; random amplified polymorphic DNA; sequence characterized amplified region marker; multiplex PCR

Artemisia plants, particularly A. iwayomogi, A capillaris, $A$. princeps, and $A$. argyi, are important medicinal materials that are utilized in traditional Asian medicines. ${ }^{2)} A$. iwayomogi and A. capillaris, referred to in Korea as 'Haninjin' and 'Injinho', respectively, ${ }^{2)}$ have been utilized traditionally for the treatment of diuresis and as anti-inflammatory agents, as is also the case in China and Japan. ${ }^{2,3)}$ Meanwhile, both $A$. princeps and $A$. argyi are referred to as 'Aeyup,' and are employed in the treatment of colic pain, vomiting, and irregular uterine bleeding. ${ }^{2,4)}$

In traditional Korean medicine markets, a portion of certain specific Artemisia herbs tend to be distributed interchangeably with other Artemisia herbs. ${ }^{2}$ For instance, some 'Haninjin' is distributed under the name 'Aeyup.' However, 'Aeyup' may occasionally be substituted with 'Haninjin' or 'Injinho' in medicinal prescriptions. To address this problem, an efficient method for the identification of Artemisia herbs is clearly necessary. Currently, the discrimination of one medicinal herb from another is a process which involves subjective methods, frequently predicated on the morphological features of the plant and/or the observations of a trained individual. However, these methods are not always sufficient for the identification of plants that morphologically resemble each other, particularly when the plants are in a dried and/or sliced state, the common forms of plants when purveyed in an herbal market. Therefore, objective methods, such as those predicated on differences in DNA sequences between organisms, ${ }^{5}$ should be developed. In a previous work, ${ }^{6)}$ we showed that a sequence-characterized amplified region (SCAR) marker ${ }^{7)}$ based on the nucleotide sequence of a specific random amplified polymorphic DNA (RAPD) product amplified uniquely from 'Aeyup' (both $A$. princeps and $A$. argyi) proved to be a reliable DNA marker for the discrimination of 'Aeyup' from other Artemisia herbs. As some Artemisia herbs can be well-distinguished from each other by RAPD, a SCAR marker of 'Haninjin' (A. iwayomogi) developed by the same strategy used for the SCAR markers of 'Aeyup' might facilitate the discrimination of 'Haninjin' from other Artemisia herbs, particularly 'Injinho' and 'Aeyup.' In this work, we developed SCAR markers of Artemisia herbs, including 'Haninjin.' We then applied these SCAR markers to the development of a method that could be applied not only to identify tested Artemisia herbs as 'Haninjin' ( $A$. iwayomogi), but also to determine which Artemisia herbs are being tested concurrently with $A$. iwayomogi.

Using this method, we were able to differentiate 'Haninjin' from other Artemisia herbs with more certainty as compared with a technique predicated on the amplification of a single DNA marker, such as a SCAR marker. Furthermore, we were able to apply this method to a search for Artemisia herbs that are substituted for 'Haninjin' and/or mixed with 'Haninjin'. In service of these research purposes, we developed a multiplex PCR method ${ }^{8)}$ using a primer set to amplify a SCAR marker of 'Haninjin', along with two other primer sets to amplify the 'Injinho' and 'Aeyup' SCAR markers, in order to amplify each SCAR marker of the Artemisia herbs in a single 
PCR process.

\section{MATERIALS AND METHODS}

Plant Materials Seventeen samples of Artemisia herbs were obtained, and are shown in Table 1. These include five samples of $A$. iwayomogi, five samples of $A$. capillaris, three samples of $A$. princeps, two samples of $A$. argyi, and one each of $A$. japonica and A. keiskeana.

The Rural Development Administration (RDA) of the Republic of Korea supplied fresh leaves of Artemisia herbs, which were acquired within the country. One sample of $A$. argyi was purchased in the Guangxi province of China. The authenticity of the samples was confirmed by the National Institute of Crop Science of the RDA of the Republic of Korea, and the samples were deposited at the Korea Institute of Oriental Medicine (KIOM).

Preparation of Genomic DNA The genomic DNA of each sample was extracted in accordance with the instruction manual for the PureGene DNA purification kit (Gentra, U.S.A.). In order to improve the DNA quality, phenolic compounds and polysaccharides were removed with $10 \%$ cetyltrimethyl ammonium bromide (CTAB) and $0.7 \mathrm{M} \mathrm{NaCl}$.

PCR Amplification The PCR for RAPD was conducted in accordance with the method described by Williams et al. ${ }^{9}{ }^{9}$ using a T-personal cycler (Biometra, Germany). In brief, 600 nM UBC primer (University of British Columbia, Canada), $1 \mathrm{U} \mathrm{Taq}$ polymerase (ABgene, U.S.A.), and $50 \mathrm{ng}$ of genomic DNA extracted from each sample were utilized for PCR amplification. The PCR process consisted of 36 cycles of the following: pre-denaturation for $5 \mathrm{~min}$ at $94^{\circ} \mathrm{C}$, denaturation for $30 \mathrm{~s}$ at $94^{\circ} \mathrm{C}$. The annealing process was conducted for $30 \mathrm{~s}$ at $37^{\circ} \mathrm{C}$, and the extension process was conducted for $1 \mathrm{~min}$ at $72^{\circ} \mathrm{C}$. A final $10 \mathrm{~min}$ reaction step was conducted at $72^{\circ} \mathrm{C}$

To amplify the SCAR markers of Artemisia herbs with the designed primer sets, PCR was conducted under identical conditions for RAPD amplification, with the exception of the annealing process conducted at $55^{\circ} \mathrm{C}$.

Multiplex PCR was also conducted using a T-personal cy- cler (Biometra, Germany). Then, $0.8 \mathrm{pmol}$ of $2 \mathrm{~F} 1 / 2 \mathrm{~F} 3$ primers, $4 \mathrm{pmol}$ of $354 \mathrm{UF} 3 / 354 \mathrm{UR} 3$ primers, $0.08 \mathrm{pmol}$ of $\mathrm{Fb} / \mathrm{R} 7$ primers, $2.5 \mathrm{U}$ of $\mathrm{Taq}$ polymerase (ABgene, U.S.A.), and $10 \mathrm{ng}$ of genomic DNA extracted from each of the Artemisia samples were used for amplification. Next, $0.16 \mathrm{pmol}$ of an AYF (5'-ACGGATATCTCGGCTC-3')/AYR (5'-GAACCATCGAGTTTTTGAAC-3') primer set, which was designed on the basis of the partial nucleotide sequences of the $5.8 \mathrm{~s}$ rDNA of Arabidopsis, was utilized to amplify the $95 \mathrm{bp}$ PCR product as an internal standard that could be used to determine the efficiency of PCR amplification. In the multiplex $\mathrm{PCR}$, pre-denaturation was conducted for $12 \mathrm{~min}$ at $95^{\circ} \mathrm{C} .30$ PCR cycles were conducted under the following conditions. Denaturation was conducted for $30 \mathrm{~s}$ at $95^{\circ} \mathrm{C}$, annealing was conducted for $30 \mathrm{~s}$ at $50^{\circ} \mathrm{C}$, and a final 30 -s extension step was conducted at $72^{\circ} \mathrm{C}$. Finally, the PCR reaction was allowed to continue for an additional $7 \mathrm{~min}$ at $72^{\circ} \mathrm{C}$. After the amplified products were separated on $1.5 \%$ agarose gel, the gel was stained with EtBr (Sigma, U.S.A.). The amplified products were then analyzed using MyImage (Seoulin Biotechnology, Republic of Korea).

Nucleotide Sequencing of PCR Products PCR products separated from the agarose gel were cloned with pGEM T-easy vector (Promega, U.S.A.). Each PCR product was amplified twice via independent PCR processes. The nucleotide sequences of the cloned PCR products were determined using two samples isolated from different colonies by Bionex (Republic of Korea).

\section{RESULTS}

Artemisia Herbs Are Discriminated through RAPD Analysis SCAR markers based on the unique RAPD-amplified PCR products of A. iwayomogi ('Haninjin') and other Artemisia herbs must be developed prior to the development of a multiplex PCR method that can be applied for the discrimination of 'Haninjin' from other Artemisia herbs, such as 'Injinho' and 'Aeyup.' Therefore, we initially attempted to ascertain whether A. iwayomogi and other Artemisia herbs could be discriminated by RAPD. On the basis of the poly-

Table 1

\begin{tabular}{|c|c|c|c|c|c|}
\hline $\begin{array}{l}\text { Medicine } \\
\text { name }\end{array}$ & $\begin{array}{l}\text { Scientific } \\
\text { name }\end{array}$ & Locality & Nationality & $\begin{array}{c}\text { Date of } \\
\text { collection }\end{array}$ & Lane \\
\hline \multirow[t]{5}{*}{ Aeyup } & \multirow[t]{3}{*}{ A. princeps } & Bongwha & Korea & 2002.9 & 1 \\
\hline & & Jeonju & Korea & 2002.5 & 2 \\
\hline & & Uiseong & Korea & 2002.9 & 3 \\
\hline & \multirow[t]{2}{*}{ A. argyi } & Suwon & Korea & 2002.6 & 4 \\
\hline & & Gvangjsih & China & 2002.7 & 5 \\
\hline \multirow[t]{5}{*}{ Injinho } & \multirow[t]{5}{*}{ A. capillaris } & Jeonju & Korea & 2002.5 & 6 \\
\hline & & Jinan & Korea & 2002.5 & 7 \\
\hline & & Jinan & Korea & 2002.5 & 8 \\
\hline & & Ullungdo & Korea & 2002.7 & 9 \\
\hline & & Bonghwa & Korea & 2002.9 & 10 \\
\hline \multirow[t]{5}{*}{ Haninjin } & \multirow[t]{5}{*}{ A. iwayomogi } & Jecheon & Korea & 2002.7 & 11 \\
\hline & & Uiseong & Korea & 2002.9 & 12 \\
\hline & & Jinan & Korea & 2002.5 & 13 \\
\hline & & Jinan & Korea & 2002.5 & 14 \\
\hline & & Bonghwa & Korea & 2002.9 & 15 \\
\hline \multirow[t]{2}{*}{ Others } & A. japonica & Suwon & Korea & 2002.6 & 16 \\
\hline & A. keiskeana & Uiseong & Korea & 2002.9 & 17 \\
\hline
\end{tabular}


morphisms revealed by 14 non-specific UBC primers, we assessed the phylogenetic similarity among the Artemisia herbs listed in Table 1 . We also conducted a phenogram by conducting UPGMA (unweighted pair-group method with arithmetic average) using the NTSYS (numerical taxonomy and multi-analysis system) program. The phenogram constructed in this work (data not shown) was almost identical to a previous phenogram predicated on 11 non-specific UBC primers revealing polymorphisms among Artemisia herbs. ${ }^{6)}$ In both phenograms, all five of the A. iwayomogi samples were categorized in one group, whereas the other Artemisia herbs were allocated to the other group. In the other group, samples of both $A$. princeps and $A$. argyi ('Aeyup') were classified into one subgroup, and samples of both $A$. capillaris and $A$. japonica were allocated into the other subgroup. However, the position of $A$. keiskeana, which was clearly separated from other Artemisia herbs, differed substantially in the two phenograms. Whereas, in a new phenogram, A. keiskeana was positioned on the other group including 'Aeyup' and both $A$. capillaris and $A$. japonica as a separated subgroup, in a previous study, A. keiskeana was positioned on one main branch of the phenogram that was separated from the other main branch, which included all samples of Artemisia herbs with the exception of $A$. keiskeana.

These results suggested that PCR product(s) amplified uniquely from $A$. iwayomogi and other Artemisia herbs might indeed be applicable to the discrimination of $A$. iwayomogi from other Artemisia constituents.

Development of a SCAR Marker of $A$. iwayomogi Among the non-specific primers revealing polymorphisms among the Artemisia herbs, primer 391 (5'- GCG AAC CTC G-3') generated $700-720 \mathrm{bp}$ PCR products from all of the A. iwayomogi samples (Fig. 1). While the RAPD method is sensitive to PCR conditions, the reproducibility of RAPD results tends to be relatively low. In order to deal with this problem, sequence-characterized amplified region (SCAR) markers were first suggested by Paran and Michelmore. ${ }^{7)}$ Therefore, based on the nucleotide sequences of these products, we attempted to develop a SCAR marker for $A$. iwayomogi. For the determination of the nucleotide sequences of these PCR products, we sub-cloned each PCR product amplified from each sample of $A$. iwayomogi (Lanes $11-15$ in Table 1). According to the determined nucleotide sequences of these PCR products, $707 \mathrm{bp}, 714 \mathrm{bp}, 718 \mathrm{bp}$, and $719 \mathrm{bp}$ PCR products were amplified and sub-cloned from lanes 11 and $15,14,13$, and 12, respectively. The nucleotide sequences of the 707, 714, 718, and 719bp PCR products were registered as AY866423, AY866420, AY866422, and AY 866421, respectively, in the GenBank database at the National Center for Biotechnology Information (NCBI). The similarities of the nucleotide sequences among three of these products, excluding the $707 \mathrm{bp}$ PCR product, were all in excess of $97 \%$. When the $707 \mathrm{bp}$ regions in the nucleotide sequences of each of the three PCR products were compared with that of the $707 \mathrm{bp}$ PCR product, similarities of 88 to $98 \%$ were noted. Based on the nucleotide sequences in which the similarities among PCR products were high, some primer sets were designed to amplify the SCAR marker of $A$. iwayomogi. Among them, 2F1 (5'-ACC TCG GAC CTA AAT ACA-3')/2F3 (5'-TTA TGA TTC ATG TTC AAT TC-3') was shown to amplify both the 364 and 365 bp PCR prod-

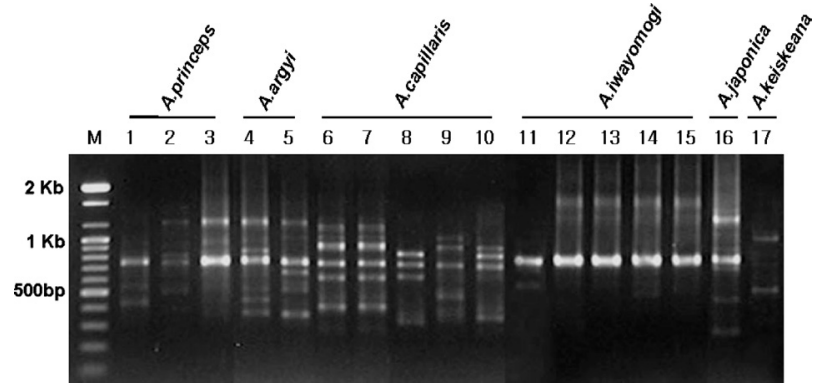

Fig. 1. Polymorphism among Artemisia Herbs Detected by Non-specific UBC Primer 391

Lanes 1-17, Artemisia herbs listed in Table 1. M, 100 bp ladder.

ucts, which appeared as a single band on the gel from $A$. iwayomogi (Fig. 3). Among them, the 2F1 (5'-ACC TCG GAC CTA AAT ACA-3')/2F3 (5'-TTA TGA TTC ATG TTC AAT TC-3') primer set designed to concurrently amplify both the 364 and 365 bp SCAR markers (Fig. 2) was also shown to amplify the PCR product, which appeared as a single band on the gel from all of the A. iwayomogi samples (Fig. 3). Using capillary electrophoresis, we confirmed that these two predicted SCAR markers were concurrently amplified from all $A$. iwayomogi samples (data not shown). Meanwhile, no PCR products were generated by this primer set in other Artemisia herbs. According to these results, the 2F1/ 2F3 primer set was capable of amplifying the SCAR marker that distinguished A. iwayomogi from other Artemisia herbs.

Development of a Common SCAR Marker of Both $A$. capillaris and $A$. japonica As previously mentioned, 'Injinho' (A. capillaris) is one of the Artemisia herbs that must be discriminated clearly from $A$. iwayomogi. Therefore, we also attempted to develop a SCAR marker for $A$. capillaris. However, as shown in a previous study, $\left.{ }^{6}\right)$ both $A$. capillaris ('Injinho') and $A$. japonica are classified into Subgroup IAb of the phenogram. Because of the phylogenetic similarity between the two Artemisia herbs, we attempted to develop a common SCAR marker of both $A$. capillaris ('Injinho') and A. japonica. In the previous study, $\left.{ }^{6}\right)$ we determined that the non-specific UBC primer 354 (5'-CTA GAG GCC G-3') specifically generated a PCR product of approximately $600 \mathrm{bp}$ from both $A$. capillaris and A. japonica. In this study, we ascertained the nucleotide sequences of these $600 \mathrm{bp}$ PCR products from both A. capillaris and A. japonica (Fig. 4). These nucleotide sequences have been registered in the NCBI GenBank database as EF204532 and ER893952, respectively. We noted an $87 \%$ similarity between the sequences of the PCR products from $A$. capillaris and $A$. japonica. Based on the established nucleotide sequences, we designed primer sets to concurrently amplify a SCAR marker common to both $A$. capillaris and $A$. japonica. Finally, we selected one primer set, 354UF3 (5'-CTA GAG GCC GAC GCG GAC-3')/354UR3 (5'-ATG CTT TTG GCT ATA TGC AGT C-3'), in order to amplify a 396 bp SCAR marker common to both $A$. capillaris and A. japonica (Fig. 5).

Development of a Multiplex PCR Method for the Discrimination of $A$. iwayomogi from Other Artemisia Herbs and for the Determination of Which Artemisia Herbs Are Being Tested In the previous study, ${ }^{6}{ }^{6}$ we developed an $\mathrm{Fb} / \mathrm{R} 7$ primer set that amplified a 254 bp SCAR marker common to both $A$. princeps and $A$. argyi ('Aeyup'). Along with 

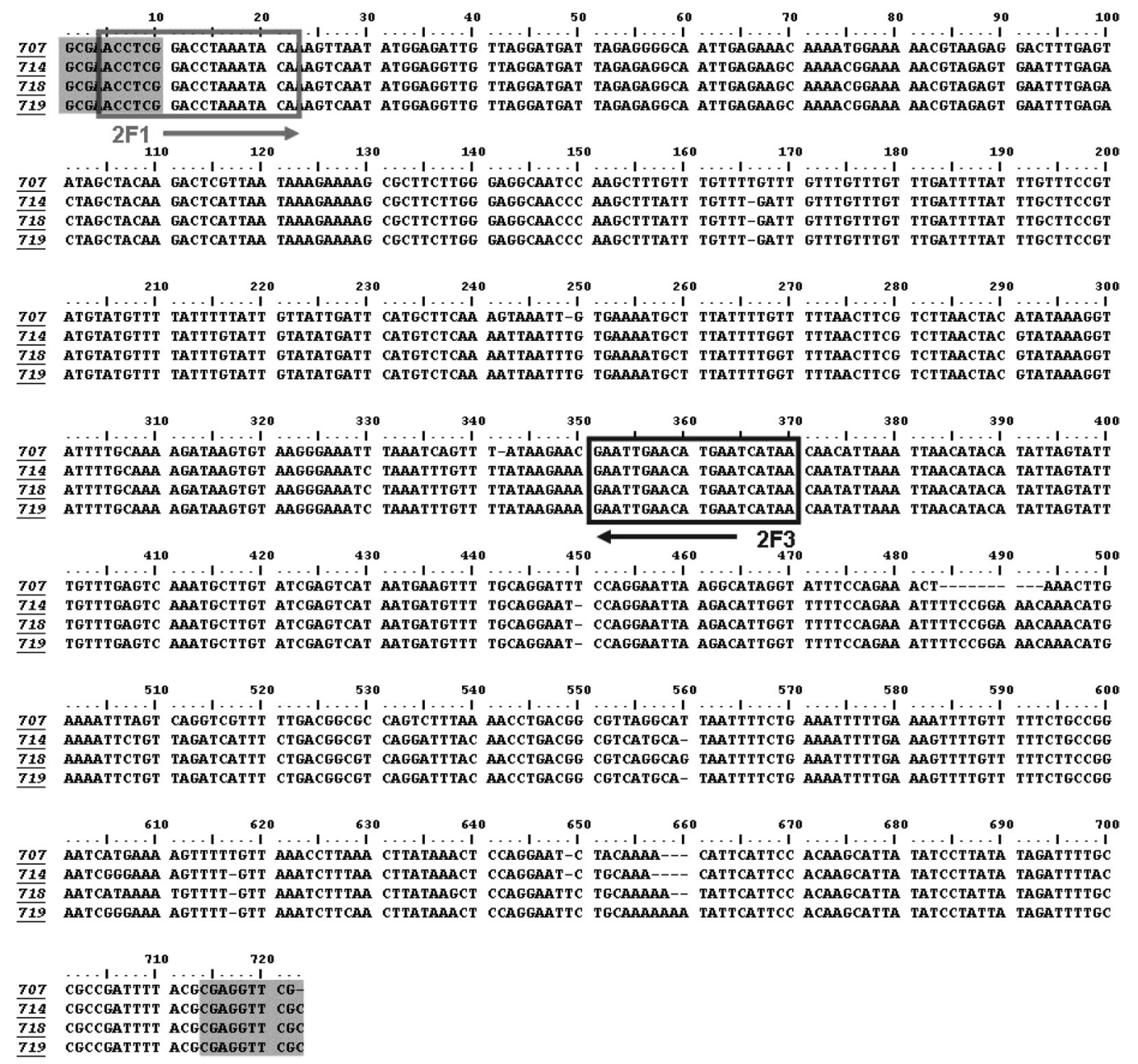

Fig. 2. Comparison of the Nucleotide Sequences of PCR Products of A. iwayomogi Amplified Using UBC Primer 391

Bold boxes around nucleotides indicate a designed primer set. Nucleotides with a highlighted background are matching sequences of UBC primer 391.

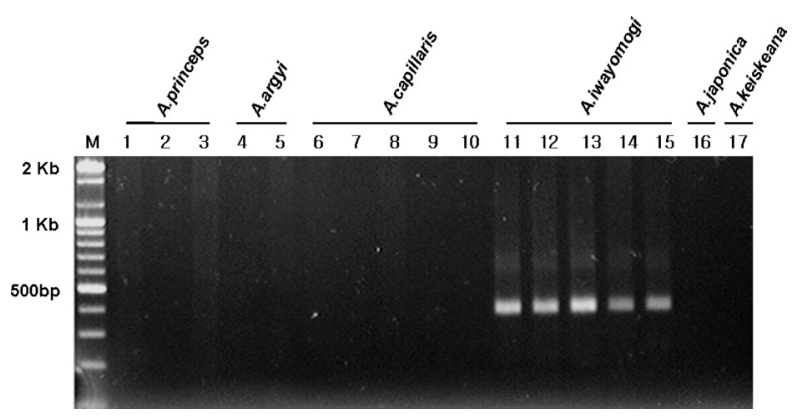

Fig. 3. PCR Products of the Designed Primer Set $(2 \mathrm{~F} 1 / 2 \mathrm{~F} 3)$ from 17 Kinds of Artemisia Herbs

Lanes 1-17, Artemisia herbs listed in Table 1. M, 100 bp ladder.

this primer set, the $2 \mathrm{~F} 1 / 2 \mathrm{~F} 3$ and $354 \mathrm{UF} 3 / 354 \mathrm{UR} 3$ primer sets were applied to the development of a multiplex PCR method that could be used to discriminate 'Haninjin' ( $A$. iwayomogi) from some other Artemisia herbs, and to concurrently determine which Artemisia herbs were tested along with A. iwayomogi. As can be seen in Fig. 6, while both the 364 and 365 bp PCR products appeared solely in 'Haninjin' (A. iwayomogi), the $254 \mathrm{bp}$ and $394 \mathrm{bp}$ PCR products were generated in the 'Aeyup' and 'Injinho' samples. As had been expected, the 354UF3/354UR3 primer sets also yielded a 394 bp PCR product in A. japonica. Meanwhile, no amplification products were generated in A. keiskeana, a member of Subgroup IB, after multiplex PCR. Therefore, this multiplex PCR method might be applicable to the discrimination of $A$. iwayomogi from other Artemisia herbs. Furthermore, this method could also be used to determine which Artemisia herbs are present in a sample, or at least into which subgroup(s) the Artemisia herbs tested along with A. iwayomogi should be classified.

\section{DISCUSSION}

For centuries, 'Haninjin' (A. iwayomogi) has been used as a traditional medicine in Korea. ${ }^{3,10)}$ In particular, aqueous 'Haninjin' extract is frequently prescribed as a crude drug for the treatment of a variety of liver diseases. ${ }^{11)}$ Recently, other pharmacological effects of $A$. iwayomogi have been observed. A. iwayomogi extract has been shown to inhibit immediate-type allergic reactions and inflammatory cytokine secretion. ${ }^{12)}$ Furthermore, a carbohydrate fraction purified from a crude water-soluble $A$. iwayomogi extract was demonstrated to suppress artificially-induced mouse thymocyte apoptosis via the modulation of Fas gene expression, which plays important roles in the damage induced by dioxin, as well as in some diseases. ${ }^{13)}$ While both 'Haninjin' (A. iwa- 


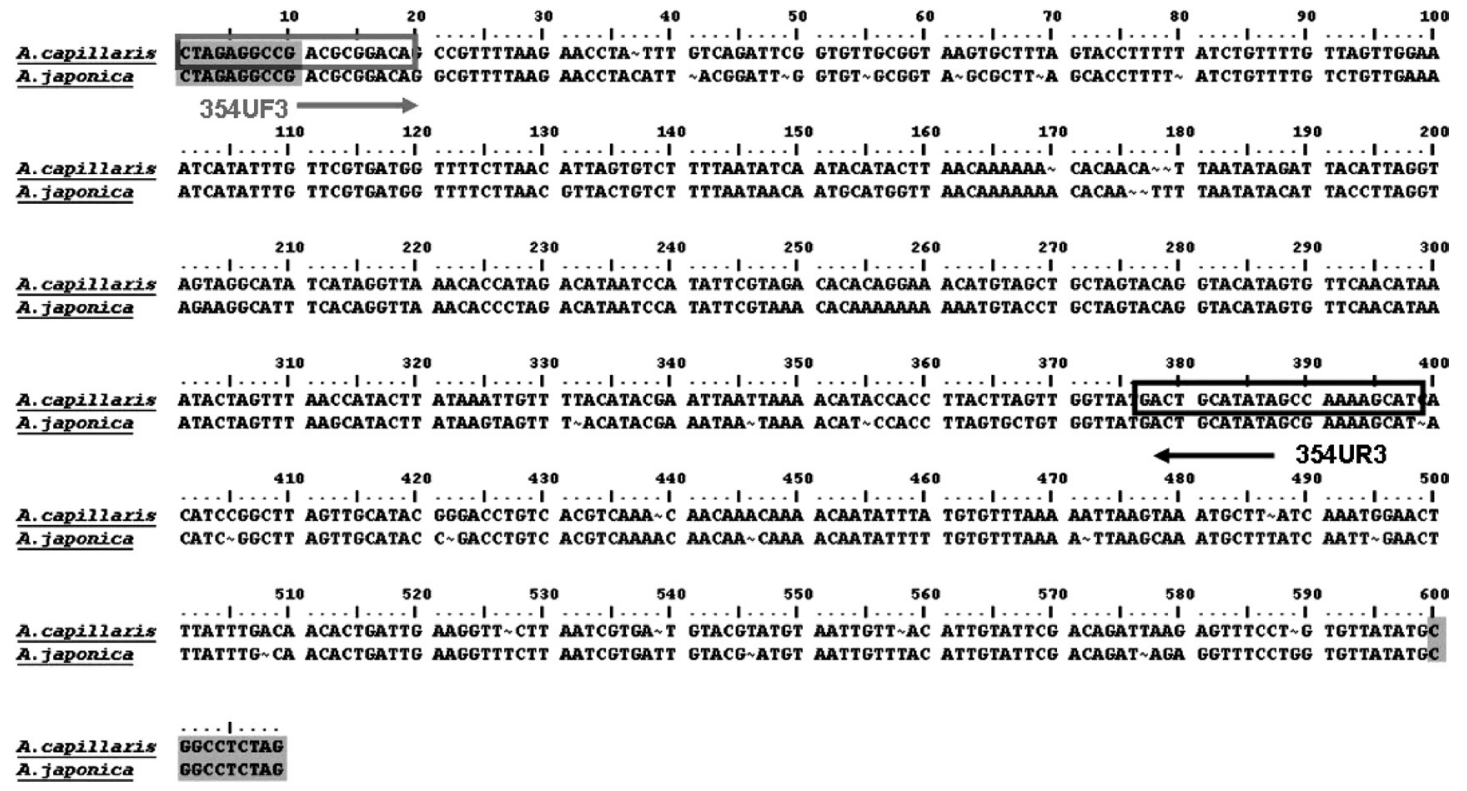

Fig. 4. Comparison of the Nucleotide Sequences of PCR Products of A. capillaris and A. japonica Amplified Using UBC Primer 354 Bold boxes around nucleotides indicate a designed primer set. Nucleotides with a highlighted background are matching sequences of UBC primer 354

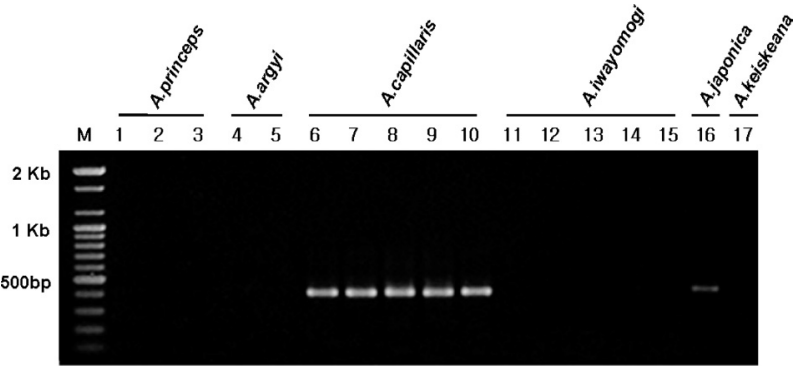

Fig. 5. PCR Products of the Designed Primer Set (354UF3/354UR3) from 17 Kinds of Artemisia Herbs

Lanes 1-17, Artemisia herbs listed in Table 1. M, 100 bp ladder.

yomogi) and 'Injinho' (A. capillaris) are often prescribed for specific diseases or symptoms, 'Haninjin' is strictly differentiated from 'Injinho' in the Republic of Korea. ${ }^{2)}$ Occasionally, 'Haninjin' and 'Injinho' are used in lieu of 'Aeyup' for medicinal prescriptions. Therefore, in this study, we have attempted to develop objective methods predicated on the relevant genetic information in order to discriminate 'Haninjin' from other Artemisia herbs, particularly 'Injinho' (A. capillaris) and 'Aeyup' (A. princeps and $A$. argyi). In service of this objective, we attempted to devise a multiplex PCR method as a more certain and useful discrimination method on the basis of the nucleotide sequences of some polymorphic PCR products of the Artemisia herbs after RAPD.

A prerequisite for this approach is that the A. iwayomogi samples must first be classified into the same group or subgroup in the phenogram of Artemisia herbs constructed on the basis of the RAPD results. In addition, other Artemisia samples must be classified into other group(s) or subgroup(s) into which A. iwayomogi samples have yet to be classified. In the previous study, ${ }^{6}$ a phenogram constructed on the basis of polymorphisms revealed through 11 primers was employed as the basis for the development of a SCAR marker of 'Aeyup' (both A. princeps and A. argyi). Because the reproducibility of RAPD is somewhat dubious, we constructed a

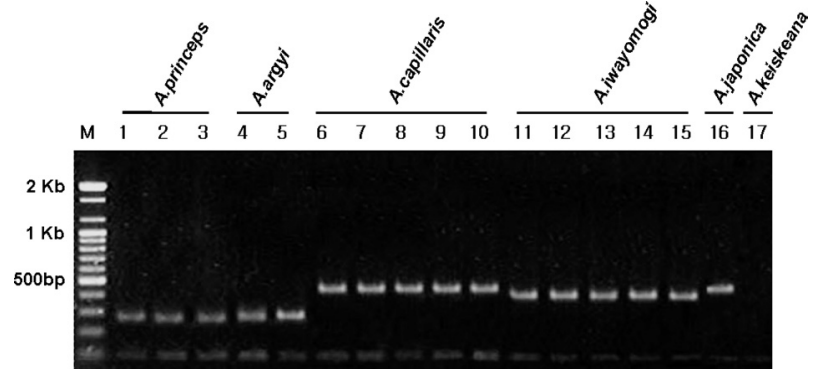

Fig. 6. Multiplex PCR Products Using Three Primer Sets $(2 \mathrm{~F} 1 / 2 \mathrm{~F} 3$, 354UF3/354UR3, Fb/R7) from 17 Kinds of Artemisia Herbs

Lanes 1-17, Artemisia herbs listed in Table 1. M, 100 bp ladder.

new phenogram predicated on the polymorphisms revealed by 14 non-specific UBC primers, including primers 303 (5'GCG GGA GAC C-3'), 321 (5'-ATC TAG GGA C-3'), and 323 (5'-GAC ATC TCG C-3'), in order to confirm the separation of Artemisia herbs on the phenogram. According to a new phenogram constructed in this study, all 'Haninjin' samples were classified into the same group. In contrast, samples of 'Injinho' (A. capillaris) and 'Aeyup' are classified into other groups. Furthermore, these two Artemisia herbs are classified into different subgroups. As shown in the new phenogram, A. japonica is classified into a subgroup that includes A. capillaris. This phylogenetic similarity between A. capillaris and A. japonica was also noted in the previous study. ${ }^{6}$ Therefore, the phenogram became the basis of the development of SCAR markers for A. iwayomogi, and for both A. capillaris and A. japonica. Furthermore, this phenogram was applied for the development of a multiple PCR method.

Four PCR products from A. iwayomogi were simultaneously amplified using primer 391 (Fig. 2), and one PCR product was amplified from both A. capillaris and A. japonica using primer 354 (Fig. 4). The nucleotide sequences of these PCR products were used to design primer sets for the amplification of each of the SCAR markers. These nu- 
cleotide sequences were compared with the sequences in the NCBI database in order to ascertain whether or not the PCR products harbored certain ORFs (open-reading frames). $230-250 \mathrm{bp}$ fragments of the $707 \mathrm{bp}$ PCR product were found to bear a similarity of $60 \%$ with the cDNAs cloned from Oryza sativa (CI319338) and Brassica napus (CD841471), the functions of which have yet to be elucidated. In addition, $220-415 \mathrm{bp}$ fragments of the 714,718 , and $719 \mathrm{bp}$ PCR products evidenced 50-60\% similarity with the cDNAs cloned from Antirrhinum majus (AJ808326) and Chlamydomonas reinhardtii (BG854434), the functions of which also have yet to be determined. However, the $270 \mathrm{bp}$ fragment of these three PCR products evidences $55-60 \%$ similarity with the lipase class 3 family protein of Arabidopsis (AT4G00500). As mentioned in the results section, both $364 \mathrm{bp}$ and $365 \mathrm{bp}$ SCAR markers of $A$. iwayomogi based on the nucleotide sequences of $707 \mathrm{bp}$, and three of 714,718 and $719 \mathrm{bp}$ PCR products respectively (Fig. 2), were concurrently amplified in all five samples of $A$. iwayomogi (data not shown). On the basis of these results, we speculate that these four PCR products amplified by primer 391 from five $A$. iwayomogi samples could harbor at least partial ORFs of the genes, which are members of a specific multi-gene family.

From $A$. capillaris and A. japonica, which were classified into the same subgroup on the phenogram (data not shown), a $599 \mathrm{bp}$ PCR product was amplified by primer 354 . We noted an $87 \%$ similarity between the nucleotide sequences of two Artemisia herbs, as shown in Fig. 4. As this work was principally focused on the discrimination of $A$. iwayomogi, we have not attempted to further discriminate $A$. capillaris from $A$. japonica using DNA markers. The nucleotide sequence of the PCR product amplified by primer 354 from A. capillaris was compared to the sequences deposited in the NCBI database. The $211 \mathrm{bp}$ fragment of the PCR product evidenced a similarity of $63 \%$ with the cDNA; these findings were similar to those from the electron transport complex protein, rnfD, which was cloned in Eleusine coracana (EB637139). In addition, the 422 bp fragment of this PCR product evidenced $57 \%$ similarity with a cDNA found in a navel orange (Citrus sinensis) callus, the function of which remains unknown (CV713837).

Therefore, the nucleotide sequences used to design the primer could also harbor partial protein information. According to a laboratory manual, ${ }^{14)}$ there is a high theoretical probability that an 18-mer primer will anneal at only one position in the majority of eukaryotic genomes, which are composed of approximately $10^{9}-10^{10} \mathrm{bp}$. Based on this information, we designed ten primer sets ranging from 18-mer to 22-mer in order to amplify the SCAR markers applied for the discrimination of Artemisia herbs. Among them, the pair of the 18-mer, 2F1, and the 20-mer, 2F3, concurrently amplified SCAR markers of $A$. iwayomogi, with sizes of 364 and 365 bp. In Fig. 3, these two PCR products are shown as a single band on the gel. Meanwhile, for the common SCAR marker of both $A$. capillaris and A. japonica, a primer set (19-mer 354UF3 and 22-mer 354UR3) that amplified a $394 \mathrm{bp}$ product (Fig. 5) was screened among a combination of 18 primers, which ranged from 19-mer to 23-mer. Along with these two primer sets, we also developed a multiplex PCR method that could be applied to distinguish 'Haninjin' from other Artemisia herbs, particularly 'Injinho' and 'Aeyup'. In the multiplex PCR technique, the template DNA isolated from Artemisia was conserved significantly as compared to that utilized in conventional PCR. Furthermore, the process was also more efficient in terms of the time, chemicals, and enzymes required for the running of PCR to amplify each SCAR marker of the Artemisia herbs. ${ }^{8,15)}$ As is shown in Fig. 6, under the multiplex PCR conditions developed in this study, a 364-365 bp SCAR marker of $A$. iwayomogi was amplified, and was determined to be located exactly between the $254 \mathrm{bp}$ and $394 \mathrm{bp}$ bands amplified from both $A$. princeps and $A$. argyi, as well as both $A$. capillaris and $A$. japonica. These results suggested that the developed multiplex PCR method could indeed be utilized for the accurate discrimination of $A$. iwayomogi. Furthermore, this technique was appropriate for the determination of Artemisia herbs tested along with $A$. iwayomogi could be classified, or at least to which subgroups the tested Artemisia herbs could be classified.

\section{REFERENCES AND NOTES}

1) Present address; Division of East District, National Institute of Scientific Investigation; Wonju 220-805, Republic of Korea.

2) "The Korean Herbal Pharmacopoeia," Korea Food \& Drug Administration, Seoul, 2002, p. 307, 383, 461, 505.

3) Kim J. O., Kim Y. S., Lee J. H., Kim M. N., Rhee S. H., Moon S. H., Park K. Y., J. Korean Soc. Food Sci. Nutr., 21, 308-313 (1992).

4) Zhao Q. C., Kiyohara H., Yamada H., Phytochemistry, 35, 73-77 (1994).

5) McClelland M., Welsh J., PCR Methods Appl., 4, 59-65 (1994).

6) Lee M. Y., Doh E. J., Park C. H., Kim Y. H., Kim E. S., Ko B. S., Oh S. E., Biol. Pharm. Bull., 29, 629-633 (2006).

7) Paran I., Michelomore R. W., Theor. Appl. Genet., 85, 985-993 (1993).

8) Burgart L., Robinson R., Heller M., Wilke W., Iakoubova O., Cheville J., Mod. Pathol., 5, 320-323 (1992).

9) Williams J. G. K., Kubelik A. R., Livak K. J., Rafalski J. A., Tingey S. V., Nucl. Acids Res., 18, 6531-6535 (1990).

10) Lee K. R., Zee O. P., Kwak J. H., Kim Y. S., Park H. K., Koo K. A., Youn H. J., Kor. J. Pharmacogn., 24, 289-295 (1993).

11) Park E. J., Nam J. X., Kim J. Y., Kang H. C., Choi J. H., Lee S. J., Lee B. H., Kim S. J., Lee J. H., Kim Y. C., Sohn D. H., J. Pharm. Pharmacol., 52, 875-881 (2000).

12) Shin T. Y., Park J. S., Kim S. H., Immunopharm. Immunotoxicol., 28, 421-430 (2006).

13) Kim A. R., Zou Y. N., Park T. H., Shim K. H., Kim M. S., Kim N. D., Kim J. D., Bae S. J., Choi J. S., Chung H. Y., Phytother. Res., 18, 1-7 (2004).

14) Dieffenbach C. W., Dveksler G. S., "PCR Primer: A Laboratory Manual," 1 st ed., Chapter 3, Cold Spring Harbor Laboratory Press, New York, 1995, pp. 133-155.

15) Herve T., Diana R., Simon K., Hoda K., Steven L. S., Genomics, 62, $500-507$ (1999). 